\title{
Multivariate Network-Level Approach to Detect Interactions between Large-Scale Functional Systems
}

\author{
Wei Gao ${ }^{1,4}$, Hongtu Zhu ${ }^{2,4}$, Kelly Giovanello ${ }^{3,4}$, and Weili Lin ${ }^{1,4}$ \\ Department of ${ }^{1}$ Radiology, ${ }^{2}$ Biostatistics and ${ }^{3}$ Psychology and ${ }^{4}$ Biomedical Research \\ Imaging Center \\ University of North Carolina at Chapel Hill, NC 27599, USA \\ wgao@email.unc.edu
}

\begin{abstract}
The question of how large-scale systems interact with each other is intriguing given the increasingly established network structures of whole brain organization. Commonly used regional interaction approaches, however, cannot address this question. In this paper, we proposed a multivariate network-level framework to directly quantify the interaction pattern between large-scale functional systems. The proposed framework was tested on three different brain states, including resting, finger tapping and movie watching using functional connectivity MRI. The interaction patterns among five predefined networks including dorsal attention (DA), default (DF), frontal-parietal control (FPC), motor-sensory (MS) and visual (V) were delineated during each state. Results show dramatic and expected network-level correlation changes across different states underscoring the importance of network-level interactions for successful transition between different states. In addition, our analysis provides preliminary evidence of the potential regulating role of FPC on the two opposing systems-DA and DF on the network level.
\end{abstract}

\section{Introduction}

Recent development of functional connectivity magnetic resonance imaging (fcMRI) [1] has greatly improved our understanding of the brain's functional organization. Multiple functional networks including motor-sensory (MS), visual (V), dorsal attention (DA), and more recently the default (DF) networks have been delineated using this technique $[1,2]$. These findings have greatly improved our understanding of whole brain functional segregation and integration and reinforced the notion that normal brain functioning relies on coordinated activity among sets of distributed yet interacted brain regional/functional systems.

Yet, how different networks interact with each other remains elusive. Fox et al [3] looked into the interaction between the DF and DA networks and observed the existence of anti-correlation between them even during a resting state. Kelly et al [4] further revealed that the strength of this negative correlation is modulated by exogenous demands and is positively correlated with behavioral performance, underscoring the functional significance of between-network interactions. Moreover, other groups [5] have recently proposed the "network degeneration hypothesis" where they reported that neurodegeneration may be related to network-level dysfunction and 
suggested the need of developing new network-based diagnostic assays. Therefore, approaches to specifically reveal brain network-level interactions may have profound implications not only in informing normal brain function but also offering means to discern brain network dysfunction in patients with neurodegenerative diseases.

While early studies provide invaluable insights into network interaction, one potential limitation is that a correlation between two mean time courses which were derived through averaging time signals over all brain regions within a network was commonly employed to quantify network level interaction [4, 6]. This essentially ignores the inter-dependence structure within a network. Although facilitating theoretical interpretation and utilizing a straightforward computation, this simplification is prone to information loss and/or even becomes untenable when the homogeneity assumption is violated.

In this study, we aimed to develop a multivariate network-level framework capable of discerning functional network interactions by integrating several statistical tools, including canonical correlation analysis (CCA) [7], multivariate regression, and a resampling method. Compared with the existing methods, three novel features make our method suitable for network-level analysis: (i) by applying multivariate techniques, our method is free from information loss induced by averaging; (ii) through dimension normalization, our method is able to handle interactions between networks of arbitrary sizes and avoid potential biases; and (iii) leveraging multiple regression, our method is not only able to study network-level correlation but also partial correlation, adding another dimension to functional analysis.

Five networks, including the mentioned MS, V, DA, DF, and another more recently delineated frontal parietal control (FPC) network [8] were included to test the proposed method. Besides, three experimental states, including resting, finger tapping and movie watching were examined to (i) detect the dynamics of network-level correlation and (ii) test the hypothesis of the FPC's regulation role during different cognitive states. Results showed extensive network-level correlation changes for successful transition between different states and provided preliminary evidence of FPC's regulating role on the two opposing systems, the DA and DF.

\section{Methods}

We develop a multivariate network-level approach for directly quantifying the correlation/partial correaltion pattern between large-scale functional systems. The overall flow chart of the proposed method is illustrated in Figure 1.

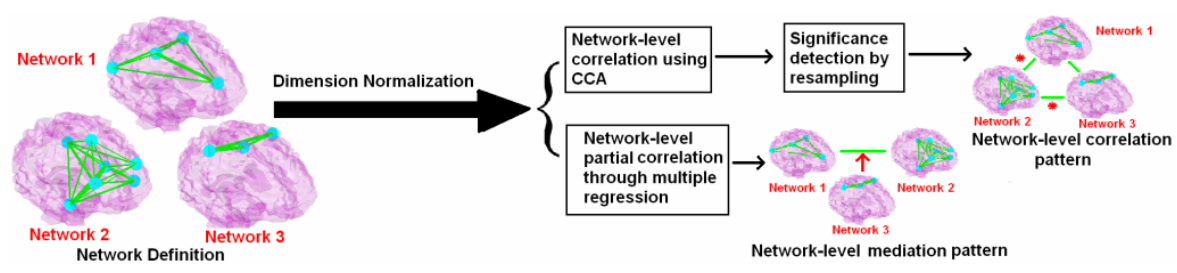

Fig. 1. Flow chart of the multivariate network-level framework 


\subsection{Multivariate Network Correlation Using Canonical Correlation Analysis}

Time courses from individual regions within each network were averaged first to represent the temporal characteristic of the whole network. Given the obvious dimension differences among different networks, there is a potential bias in the network-level interaction calculation. To overcome this bias, principle component analysis (PCA) was performed and the top 4 principle components for each network were selected for subsequent multivariate calculations. A retrospective check reveals that more than $98 \%$ percent of the variance is preserved for all networks, but the number of components can be adjusted in a study-dependent manner.

To quantify the network level interaction, a canonical correlation measure between two multivariate vectors was used. Canonical correlation analysis [7] has been widely employed to measure the association between multivariate variables: $X=\left[x_{1}, \ldots, x_{m}\right]^{T}$ and $Y=\left[y_{1}, \ldots, y_{n}\right]^{T}$. Particularly, for two univariate random variables, the canonical correlation is identical to the Pearson's correlation. The key idea of CCA is to maximize the correlation between linear combinations of $X$ and $Y$ denoted by $U_{1}=w_{1} x_{1}+\ldots+w_{m} x_{m}=w^{T} x$ and $U_{2}=v_{1} y_{1}+\ldots+v_{n} y_{n}=v^{T} y$, respectively. The canonical correlation equals to the largest eigenvalue of the matrix of $C_{x x}^{-1} C_{x y} C_{y y}^{-1} C_{y x}$ (or $C_{y y}^{-1} C_{y x} C_{x x}^{-1} C_{x y}$ ). Notice the value of network canonical correlation $\left(N C C_{x y}\right)$ is between 0 and 1 with 0 indicating no dependence and 1 indicating full dependence.

\subsection{Network-Level Partial Correlation through Multivariate Regression}

In bivariate statistics, partial correlation is used to assess potential mediation effect, which is defined as the correlation between two random variables after controlling for another "mediator" variable. Specifically, partial correlation $\rho_{x y / z}$ between two random variables $x$ and $y$ controlling for another variable $z$ can be computed as the Pearson's correlation between the residuals $\varepsilon_{x}$ and $\varepsilon_{y}$ from two linear regression equations:

$$
\begin{aligned}
& x=x_{0}+\beta_{1} z+\varepsilon_{x} \\
& y=y_{0}+\beta_{2} z+\varepsilon_{y}
\end{aligned}
$$

For mediation analysis, $\rho_{x y / z}$ denotes the correlation remained between $x$ and $y$ when all mediating effect of $z$ is removed and the differences between the ordinary correlation $\rho_{x y}$ and $\rho_{x y / z}$, represent the amount of mediation that $z$ exerts on the relation between $x$ and $y$.

Since the primary focus of this study is to depict correlation between two sets of variables, the above computational procedures are generalized to accommodate the multivariate property of this problem. For two sets of multivariate vectors $X=\left[x_{1}, \ldots, x_{m}\right]^{T}$ and $Y=\left[y_{1}, \ldots, y_{n}\right]^{T}$, and another set of independent 
covariates $Z=\left[z_{1}, \ldots, z_{p}\right]^{T}$, influences from which will be removed, Eq.1 and Eq. 2 can be written as:

$$
\begin{aligned}
& X=X_{0}+\mathrm{B}_{1} Z+E_{x} \\
& Y=Y_{0}+\mathrm{B}_{2} Z+E_{y}
\end{aligned}
$$

where $E_{x}$ and $E_{y}$ are the residual vectors of $\mathrm{X}$ and $\mathrm{Y}$, respectively, after regressing on the variable set of $\mathrm{Z}$. Subsequently, the canonical correlation coefficient of $E_{x}$ and $E_{y}$ can be calculated, representing the partial correlation $N P C_{X Y} / Z$ between the two sets of variables (networks) $\mathrm{X}$ and $\mathrm{Y}$. The difference of $N C C_{X Y}-N P C_{X Y / Z}$ thus represents the potential mediation effects of $\mathrm{Z}$ exerting on the relation between $\mathrm{X}$ and $\mathrm{Y}$.

\subsection{Resampling to Define Significance of Network-Level Correlation}

After calculating network-level correlations, the next question is to determine whether a certain correlation is statistically significant. Rather than testing the null hypothesis of zero interaction using random signal, we prompt to use a null distribution derived from resampling of the actual brain signals. Specifically, this null distribution is obtained by randomly permuting a set of reference regions into two sets and calculating their network level correlations. The reference regions are chosen to be those that demonstrate the minimum possible interactions (according to the absolute values of Pearson's correlation) among each other from the 90 pre-defined brain regions covering the whole brain [9]. The rationales behind this choice are (i) the wide spread dependence (either positive or negative correlation) between different brain regions and (ii) the large number of regions involved in each network. This means that even by randomly selecting a number of regions (among the least interacted regions) to construct two networks, certain amount of dependence between them is possible, which can be reasonably defined as the "baseline" for network-level correlations. As a result, network-level interactions that are comparable to this baseline should be excluded and only those that are statistically higher should be considered.

More specifically, based on the 90 ROI template [9], we selected a set of 15 reference regions during each state, which were then randomly distributed to 2 networks with 6 and 9 regions (equal to the largest possible combination of number of regions in the five selected networks, details in Section 3) and the network-level correlations were calculated 1000 times to generate the null distribution for each state, from which the p-value for each correlation can be defined using appearance ratio. This process was done for each subject and the obtained p-values were combined across subjects to give a group p-value using the Fisher's method [10] for each correlation. Significant correlations were defined at $\alpha=0.05$ after correcting for multiple comparisons using FDR [11]. The same procedures were done for each of the three states examined. 


\section{Materials}

A total of 19 healthy subjects (age 25 33, 7F, all right-handed) were recruited in this study. A T2*-weighted EPI sequence was used to acquire fcMRI signal on a $3 \mathrm{~T}$ scanner: $\mathrm{TR}=2 \mathrm{sec}, \mathrm{TE}=32 \mathrm{~ms} ; 33$ slices; and voxel size $=4 \times 4 \times 4 \mathrm{~mm}^{3}$. This sequence was repeated 150 times $(\sim 5 \mathrm{~min})$ for each of the three states. During resting, subjects were instructed to relax and remain still but keep eyes closed. During finger tapping, subjects were instructed to continuously touching the thumb to each of the other fingers in a sequential manner at a roughly consistent pace $(\sim 1 \mathrm{~Hz})$ with eyes closed. For movie watching, the movie clip contains shallow sea scenes.

After standard preprocessing steps, including time shifting, motion correction, spatial smoothing (6-mm FWHM Gaussian kernel), and band filtering $(<0.08 \mathrm{~Hz})$, nuisance sources of variance (white matter, CSF and the mean global signal) were removed through regression. Three subjects were excluded from the subsequent analysis because of excessive head motion. Images of the first ten time points were excluded to allow magnetization reaching a steady state. Spatial normalization to MNI template was achieved using nonlinear registration and regional parcellation was done base on Tzourio-Mazoyer et al [9].

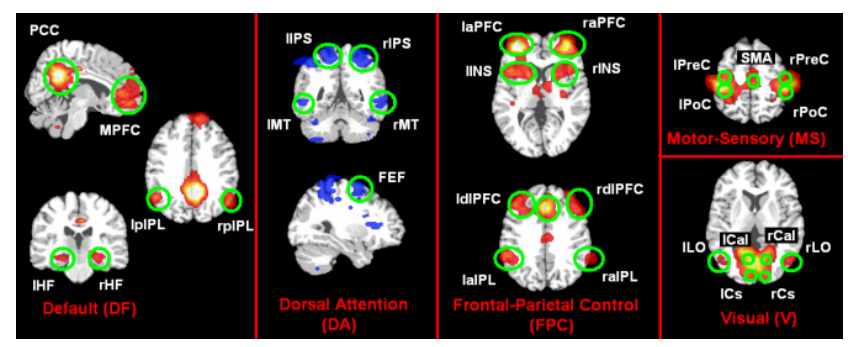

Fig. 2. ROIs defined for the five networks based on functional correlation map

The network definition is similar as that done by Van Dijk et al [2], which is based on the combined consideration of (i) prior knowledge of network composition and (ii) the study-specific functional correlation map. Specifically, regions of interest (ROIs) were defined as 8-mm spheres around the detected peaks (high correlation values) to construct the corresponding networks. For peak definition, an initial seed in the posterior cingulate cortex (PCC) was used to compute a group mean correlation map which was then used to indentify peaks within the DF network, including the medial prefrontal cortex (MPFC), bilateral inferior parietal lobule (IPL) and hippocampus formation (HF). Given the well-established anti-correlation with DF [3], the negative peaks of the PCC correlation map were identified for regions in the DA network, including the bilateral intra-parietal sulcus (IPS), frontal eye field (FEF) and middle temporal area $(\mathrm{MT}+)$. For FPC, the seeds were centered at the bilateral aPFC [8] to define peaks in nodes, including the anterior cingualte cortex (ACC), dorsolateral prefrontal cortex (dlPFC), anterior insula (aINS), and anterior inferior parietal lobule (aIPL). In addition, the MS and V networks were similarly defined using the bilateral postcentral and calcarine cortex as initial seeds, respectively, to define peaks in 
precentral/postcentral gyrus (PreC, PoC), supplementary motor-sensory area (SMA) for MS and bilateral calcarine (Cal), cuneus (CS), and lateral occipital (LO) for V. Note all functional network definitions were based on the resting state data and altogether there are 6, 9, 6, 5, and 6 regions for DA, FPC, DF, MS and V, respectively, which are presented in Fig. 2.

\section{Experimental Results}

The network-level correlation patterns using the spring-embedding plots are presented in Fig.3. The width of edges corresponds to the strength of interactions. Moreover, the statistical grouping of the set of interaction values are also achieved using the Tukey's test at $\alpha=0.05$ to detect those that are significantly higher than the rest during each state (red asterisks in Fig.3).

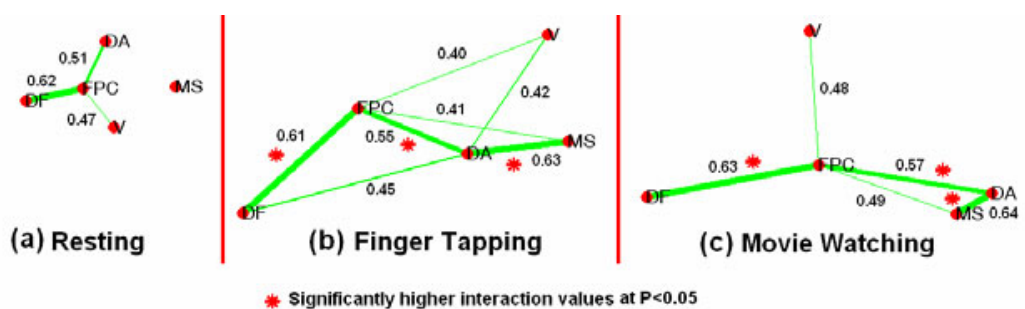

Fig. 3. The network-level correlation pattern during the three examined states. The value besides each connection represents the group average network-level correlation. Red asterisks indicate statistically stronger correlations using Tukey's test at $\alpha=0.05$.

During the resting state, only three significant connections exist with FPC at the center and connected with DA, DF, and V while MS is left alone. This sparse structure shows minimal interactions among networks during resting but the center position of FPC implies its significant "bridging" role, which will be specifically tested later. During finger tapping, the interaction pattern becomes much more extensive: besides the observed 3 interactions during resting, DA now significantly interacts with MS, which is highly expected given the apparent attentional requirement. In addition, this observation could also suggest DA's top-down control over MS for successful task performance[12]. Moreover, the DA-DF also becomes significant which is consistent with the previously reported increased anti-correlation between them during goal-directed tasks [4]. Other significant interactions include DA-V, and FPC-MS. During movie watching, the changes are less extensive but still two more interactions (DA-MS, FPC-MS) emerge which might be due to the increased control over eye movement. Overall, minimal network-level interactions during resting together with the enhanced interactions during the two task states indicate increased coordination/competition between networks during task performance.

Another interesting pattern is that interactions between FPC-DA and FPC-DF persist across all three states and they are statistically stronger than other interactions 


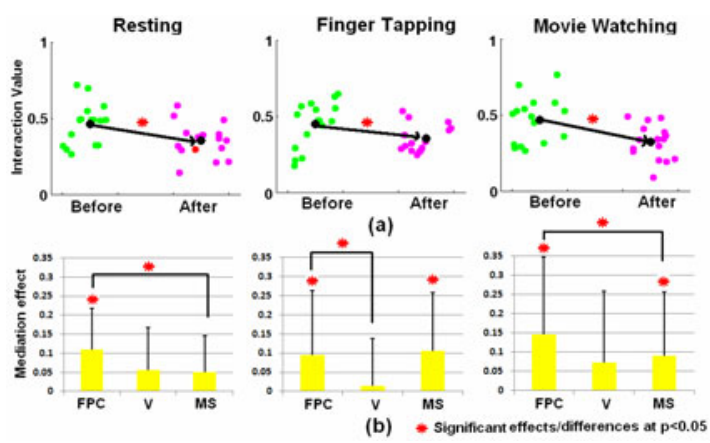

Fig. 4. Mediation analysis on the network-level interaction between DA and DF during three examined states. (a) mediation effect of FPC by comparing the interaction values (DA-DF) before (green dots) and after regressing out its effect (pink dots); (b) comparisons of FPC's mediation effect on DA-DF with that of $\mathrm{V}$ and MS across all three states.

during both finger taping and movie watching (except DA-MS, which is comparable, Fig.3). These findings clearly indicate the importance of this triad system and are consistent with the hypothesis [8] that FPC might regulate the two opposing systemsDA and DF. To directly test this hypothesis, network-level partial correlation between DA and DF using FPC as a control network was calculated during each state and the interaction values before and after this regression were compared to test the mediation role of FPC between these two systems. The results are shown in Fig.4a, where a significant reduction of interaction strength is observed for all three states $(\mathrm{p}=0.0012$, 0.0377, and 0.0105 using one way ANOVA), strongly supporting FPC's regulation role on DA and DF. Moreover, this effect (by taking the difference between values before and after regressing out of its effect) is compared among FPC, MS, and V (Fig.4b), where it shows that (i) FPC is the only network demonstrating significant correlation-reduction effect during resting; (ii) $\mathrm{V}$ fails to show any significant effect during any state; and (iii) although MS shows significant effect during the two task states, its effect is significantly weaker than that of FPC during movie watching. Note although causal relationship should be validated before concluding this regulating role, our results provide initial support for FPC's regulating role over the two most salient, anti-correlated systems- DA and DF on the network level.

\section{Conclusion}

In this paper, we proposed a new multivariate network-level framework to quantify the correlations/partial correlations between large-scale systems and demonstrated its application during three different brain states, including resting, finger tapping and movie watching. Through direct application of multivariate techniques, our method is (i) free of information loss induced by averaging; (ii) able to handle networks of arbitrary size through dimension normalization; and (iii) able to detect potential mediation effects through multiple regressions. Experimental results show dramatic and extensive network correlation changes which are consistent with our expectations 
and previous findings [8], underscoring the importance of network level coordination in task fulfillment. Moreover, network-level partial correlation analysis provides support for the potential regulating role of FPC over the two opposing systems-DA and DF.

Acknowledgement. This work was supported in part by NSF (BCS-08-26844) and NIH (NS RO1055754, UL1-RR025747-01, MH086633, P01CA142538-01 and AG033387).

\section{References}

1. Biswal, B.B., Yetkin, F.Z., Haughton, V.M., Hyde, J.S.: Functional connectivity in the motor cortex of resting human brain using echo-planar MRI. Magn. Reson. Med. 34, $537-$ 541 (1995)

2. Van Dijk, K.R., Hedden, T., Venkataraman, A., Evans, K.C., Lazar, S.W., Buckner, R.L.: Intrinsic functional connectivity as a tool for human connectomics: theory, properties, and optimization. J. Neurophysiol. 103, 297-321 (2010)

3. Fox, M.D., Snyder, A.Z., Vincent, J.L., Corbetta, M., Van Essen, D.C., Raichle, M.E.: The human brain is intrinsically organized into dynamic, anticorrelated functional networks. Proc. Natl. Acad. Sci. USA 102, 9673-9678 (2005)

4. Kelly, A.M., Uddin, L.Q., Biswal, B.B., Castellanos, F.X., Milham, M.P.: Competition between functional brain networks mediates behavioral variability. Neuroimage 39, $527-$ 537 (2008)

5. Seeley, W.W., Crawford, R.K., Zhou, J., Miller, B.L., Greicius, M.D.: Neurodegenerative diseases target large-scale human brain networks. Neuron. 62, 42-52 (2009)

6. Jafri, M.J., Pearlson, G.D., Stevens, M., Calhoun, V.D.: A method for functional network connectivity among spatially independent resting-state components in schizophrenia. Neuroimage 39, 1666-1681 (2008)

7. Hotelling, H.: Relations between two sets of variants. Biometrika, 312-377 (1936)

8. Vincent, J.L., Kahn, I., Snyder, A.Z., Raichle, M.E., Buckner, R.L.: Evidence for a frontoparietal control system revealed by intrinsic functional connectivity. J. Neurophysiol. 100(6), 3328-3342 (2008)

9. Tzourio-Mazoyer, N., Landeau, B., Papathanassiou, D., Crivello, F., Etard, O., Delcroix, N., Mazoyer, B., Joliot, M.: Automated anatomical labeling of activations in SPM using a macroscopic anatomical parcellation of the MNI MRI single-subject brain. Neuroimage 15(1), 273-289 (2002)

10. Lazar, N.A., Luna, B., Sweeney, J.A., Eddy, W.F.: Combining brains: a survey of methods for statistical pooling of information. Neuroimage 16(2), 538-550 (2002)

11. Benjamini, Y., Yekutieli, D.: The contorl of the false discovery rate in multilpe testing under dependency. Ann. Statist. 29, 1165-1188 (2001)

12. Gordon, A.M., Lee, J.H., Flament, D., Ugurbil, K., Ebner, T.J.: Functional magnetic resonance imaging of motor, sensory, and posterior parietal cortical areas during performance of sequential typing movements. Exp. Brain Res. 121(2), 153-166 (1998) 